\title{
Maternal nutritional status mediates the linkage between household food insecurity and mid-infancy size in rural Bangladesh
}

\author{
Muzi $\mathrm{Na}^{1}$, Abu Ahmed Shamim²,3,4, Sucheta Mehra ${ }^{2,3}$, Alain Labrique ${ }^{2,3}$, Hasmot Ali ${ }^{2,3}$, Lee S.-F. Wu ${ }^{2,3}$, \\ Saijuddin Shaikh ${ }^{2,3}$, Rolf Klemm ${ }^{2,3,5}$, Parul Christian ${ }^{2,3}$ and Keith P. West $\mathrm{Jr}^{2,3 *}$ \\ ${ }^{1}$ Department of Nutritional Sciences, The Pennsylvania State University, University Park, PA 16802, USA \\ ${ }^{2}$ Center for Human Nutrition, Department of International Health, Johns Hopkins Bloomberg School of Public Health, \\ Baltimore, MD 21205, USA \\ ${ }^{3}$ The JiVitA Project, Johns Hopkins University Bangladesh, Gaibandha 5700, Bangladesh \\ ${ }^{4}$ Centerfor Non-communicable Diseases and Nutrition, BRAC James P Grant School of Public Health, BRAC University, Dhaka, \\ Bangladesh \\ ${ }^{5}$ Helen Keller International, Washington, DC 20006, USA \\ (Submitted 1 October 2019 - Final revision received 10 February 2020 - Accepted 19 February 2020 - First published online 27 February 2020)
}

\section{Abstract}

Household food insecurity (HFI) is a major concern in South Asia. The pathways by which HFI may reduce child growth remain inadequately understood. In a cohort study of 12693 maternal-infant dyads in rural Bangladesh, we examined association and likely explanatory pathways linking HFI, assessed using a validated nine-item perception-based index, to infant size at 6 months. Mothers were assessed early in pregnancy for anthropometric status, dietary diversity and socio-economic status. Infants were assessed for weight, length, and arm, chest and head circumferences and breast and complementary feeding status at birth and 6 months of age. Extent of HFI shared a negative, dose-response association with all measures of infant size at 6 months and odds of wasting and stunting; 57-89\% of variances in the unadjusted models were explained by prenatal factors (maternal nutritional status and dietary diversity), and birth size adjusted for gestational age. Postnatal infant breast and complementary feeding and morbidity exposures explained the remaining fraction of the significant association between HFI and differences in infant arm and chest circumferences and odds of underweight. Contextual (i.e. socio-economic) factors finally brought remaining nonsignificant fractions of the food insecurity-related mid-infancy growth deficit to practically zero. Improving food security prior to pregnancy and during gestation would likely improve infant growth the most in rural Bangladesh.

Key words: Food insecurity: Infant growth: Bangladesh: Mediation

Food insecurity is a global concern and entrenched problem in rural South Asia, periodically amplified by seasonality, economic crises and effects of climate change ${ }^{(1)}$. In 2018, the number of undernourished people estimated by the FAO was about 820 million worldwide, with the largest fraction, $34 \%$ or 279 million, living in Southern Asia ${ }^{(2)}$. Coexisting with widely prevalent food insecurity is a high burden of preschool child stunting, affecting one-third of its young children in the Southern Asia region in $2018^{(2)}$. Among the most affected groups are children in rural Bangladesh where, based on the most recent demographic data from 2014, 36, 14 and $33 \%$ of preschoolers are stunted, wasted and underweight ${ }^{(3)}$.
Household food insecurity (HFI) could influence young child growth via several pathways, as has long been captured in the UNICEF framework for malnutrition ${ }^{(4)}$. First, HFI may influence infant growth through the maternal-fetal nutrition pathway. HFI is associated with insufficient food access to women of reproductive age which likely extends through periods of pregnancy and lactation ${ }^{(5-7)}$. Prenatal factors, such as maternal nutritional status before and during pregnancy, are a critical determinant of intra-uterine growth, birth size ${ }^{(8-10)}$ and postnatal linear growth ${ }^{(11)}$. Second, HFI could alter postnatal maternal-infant interactions ${ }^{(12)}$, which may result in changes in feeding behaviours ${ }^{(13,14)}$ and increased illness ${ }^{(15)}$

Abbreviations: GA, gestational age; HFI, household food insecurity; HFII, household food insecurity index; MUAC, mid-upper arm circumference; SES, socio-economic status.

* Corresponding author: Keith P. West, fax +1-410-955-0196, email kwest@jhsph.edu 
and mortality ${ }^{(16)}$ through complex, interacting pathways. Given the complexity of mechanisms, prospective studies that can partition prenatal, postnatal and contextual factors explaining the HFI and its nutritional consequences would enable a greater understanding of the ways in which food insecurity may affect infant and child growth.

In this study, we investigated the association between HFI and infant size and risk of malnutrition at age of 6 months using longitudinal data from a birth cohort in which we measured several prenatal, at-birth, postnatal and other contextual factors, including nutritional status of mothers early in pregnancy, birth sizes, as well as feeding practices, morbidity and maternal and household socio-economic status (SES). Our aim was to identify components and likely mechanisms explaining observed associations between HFI and infant size at 6 months of age in rural Bangladesh.

\section{Subjects and methods}

\section{Mother-infant dyads}

Subjects for this study were rural Bangladeshi mothers, with their 6-month-old infants, who participated in a large, clusterrandomised trial designed to examine the efficacy of a daily antenatal supplement, containing fifteen micronutrients, compared with folic acid and Fe use alone, on improving fetal and infant health and survival(17). The trial was undertaken in Gaibandha and Rangpur Districts, covering an area of approximately $435 \mathrm{~km}^{2}$ with a population of approximately $650000^{(18)}$. Married women of reproductive age (13-45 years) living in nineteen contiguous unions were placed under a five weekly, home-based pregnancy surveillance, during which they were asked about having menstruated in the previous month. Amenorrhoeic women were offered a urine test to confirm pregnancy and, if pregnant, consented and begun to receive study supplements on a weekly basis through 12 weeks post-partum. Usually within a week after recruitment, women were revisited at home, asked about previous pregnancy history, frequency of dietary intake of thirty-two food in the previous $7 \mathrm{~d}$, weighed lightly clothed on SECA digital scales (UNICEF) to the nearest $100 \mathrm{~g}$, measured in terms of height using a portable stadiometer and left mid-upper arm circumference (MUAC) with an insertion tape ${ }^{(19)}$, both to the nearest $0 \cdot 1 \mathrm{~cm}$. For height and MUAC, the median of triplicate measurements was taken as the representative value. Parity was counted as the number of live births prior to this pregnancy. Women's gestational age (GA) at first anthropometric measurement was calculated as the difference between the measurement date and the date of last menstrual period. Women's dietary diversity score was calculated as the total number of food groups consumed out of ten food groups ${ }^{(20)}$ in the previous week: non-rice starchy staples, dark green leafy vegetables, vitamin-A-rich fruit and vegetables, other fruit and vegetables, legumes and nuts, organ meat, meat, fish, eggs and dairy products. Maternal BMI was calculated as weight $/$ height ${ }^{2}\left(\mathrm{~kg} / \mathrm{m}^{2}\right)$. Wealth index calculated using household SES variables was based on a previously standardised methodology ${ }^{(21)}$.
A community-based birth notification system was set up to enable trained field staff to visit mothers and newborn children usually within a week after birth to assess infant size. Naked birth weight of infants was measured to the nearest $10 \mathrm{~g}$ on a TANITA BD-585 scale (Tanita Corporation); recumbent length was measured using a portable, plexiglass, folding length board with fixed head piece and sliding foot block modified from the Infant Shorr board (Shorr Productions) and head circumference, chest circumference and left MUAC measurements were taken using an Ross insertion tape (Abbott Laboratories), all to the nearest $0.1 \mathrm{~cm}$, following previously described methods ${ }^{(22)}$. GA in weeks at birth was calculated based on the interval between the dates of last menstrual period and delivery. Preterm birth is defined as $<37$ weeks of GA before delivery. At 6 months postpartum, infants were revisited to evaluate vital status, anthropometric status by the same procedures, breastfeeding frequency and sufficiency, introduction of non-breast milk foods and histories of morbidity symptoms in the previous $7 \mathrm{~d}$ including acute respiratory, diarrhoea, dysentery and fever. Added food items were reported by ten food groups ${ }^{(23)}$ : (1) infant formula; (2) milk (fresh or powdered); (3) dairy products (yogurt or other dairy products); (4) plain water; (5) any grains (suji/payesh, wheat/rice flour gruel, tapioca, rice, Khichari); (6) dal; (7) banana; (8) biscuit; (9) added oil (oil or ghee); (10) added sugar and (11) other food; Infant BMI at approximately 6 months was calculated as weight/height ${ }^{2}$ $\left(\mathrm{kg} / \mathrm{m}^{2}\right)$, whereas an infant's ponderal index at birth was calculated as weight $/$ height ${ }^{3}\left(\mathrm{~kg} / \mathrm{m}^{3}\right)$. Infant weight and length measurements were converted to weight-for-length, weight-for-age and length-for-age $z$-scores using the WHO Multicenter Growth Reference Study child growth standards, using WHO Anthro version 3.2.2 (WHO). Wasting, stunting and underweight were defined as $<-2 z$-score for weight-for-length, length-for-age and weight-for-age, respectively.

At 6 month postpartum, HFI was measured by using a nineitem Food Access Survey Tool, which was developed and tested by Food and Nutrition Technical Assistance Project in Bangladesh $^{(24)}$. Previously, we have found that HFI measured at 6 months postpartum was longitudinally associated with declined maternal dietary diversity during pregnancy and lactation in the same study population, suggesting chronic HFI in rural Bangladesh ${ }^{(7)}$. The Food Access Survey Tool reflects the concept of food security in four domains: anxiety over food acquisition, quality of food, quantity of food and social acceptability. Subjects were asked to recall the frequency of the following behaviour or concerns in the past 6 months: eating square meals, eating wheat (instead of rice), skipping meals, eating less food, having no money to buy food, worrying about food, buying rice, taking out a loan from shops or borrowing money to buy food. Responses for frequency were provided in a semiquantitative manner: $0=$ never ( 0 time $/ 6$ months); $1=$ rarely (1-3 times/ 6 months); $2=$ sometimes (4-6 times/ 6 months); $3=$ often (a few times each week) or $4=$ mostly (most days per week). Question about 'square meals' is reversely coded in order to be consistent with higher frequency for more severe food insecurity as in other questions. Sum of the responses to all nine questions were calculated as the household food insecurity 
index (HFII). Households with all 'never' responses is defined as food-secure group $(\mathrm{HFII}=0)$. The rest of households were then categorised into mild $(1 \leq \mathrm{HFII} \leq 3)$, moderate $(4 \leq \mathrm{HFII} \leq 7)$ and severe $(8 \leq$ HFII $\leq 36)$ food insecurity based on the tertile cutoffs of non-zero HFII values. Six standard seasons were defined based on HFI assessment date using the Bangladeshi calendar starting from the middle of December for every 2 months ${ }^{(25)}$.

\section{Statistical analysis}

All of the results were reported by HFI index category, where HFI is treated as categorical variables. $\chi^{2}$ tests were used to compare maternal, infant and household characteristics. Nonparametric tests for linear trend across the ordered HFI groups were applied on maternal and infants' anthropometric measures. We developed the conceptual framework (Fig. 1), hypothesising a maternal-pregnancy-fetal nutrition pathway and a maternal-infant interaction pathway through which HFI may influence infant growth. The maternal-fetal nutrition pathway features prenatal factors (e.g. maternal nutritional status at onset of pregnancy and maternal dietary diversity during pregnancy or women's dietary diversity score) and birth sizes. The maternal-infant interaction pathway features postnatal factors including feeding practices and infant morbidity. Contextual factors include several maternal and household SES factors and season. Model 0 estimated the unadjusted association. Model 1 adjusted for infant sex, age in months at 6 months follow-up, parity, maternal age and GA at enrolment. From model 2 to model 5, a set of multiple linear regression models were applied with cumulative adjustment on a temporal sequence starting from prenatal factors including prenatal factors (model 2: additionally adjusted for maternal height, MUAC and women's dietary diversity score) followed by birth size as a proxy for fetal growth outcome (model 3: additionally adjusted for infant's GA at birth, birth length and ponderal index), postnatal factors (model 4: additionally adjusted for breast and complementary feeding practices and child morbidity), and finally the contextual factors (model 5: additionally adjusted for maternal employment, education, wealth index and season). Similarly, a set of multiple logistic regression models were used

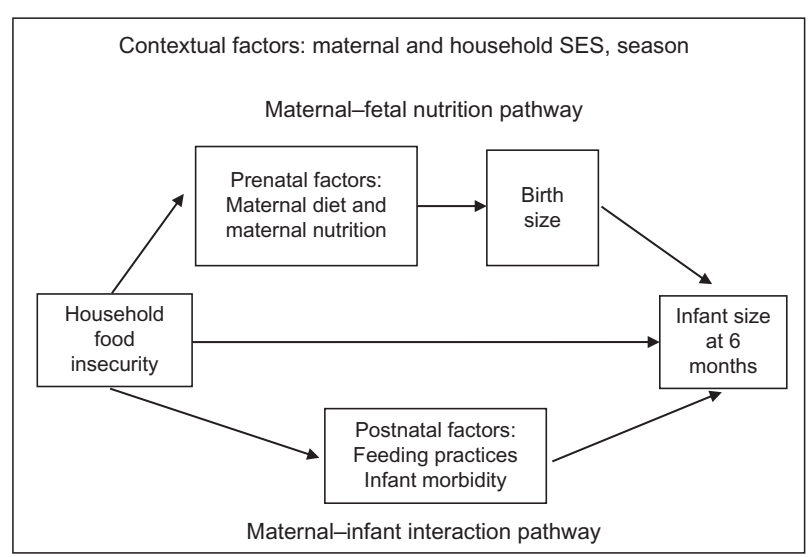

Fig. 1. Conceptual framework of the association between household food insecurity and infant size at 6 months. SES, socio-economic status. with the same adjustment procedure to study HFI and risk of wasting, stunting and underweight at 6 months of age. Feeding practices and child morbidity that were found significantly different with HFI status were included in the multiple regression models. We set the primary level of statistical significance at $P<0 \cdot 05$. All analyses were performed using R 2.13 .2 (The R Foundation for Statistical Computing).

This trial was conducted according to the guidelines laid down in the Declaration of Helsinki, and all procedures involving human subjects were approved by the Bangladesh Medical Research Council, Dhaka, and the Institutional Review Board of the Johns Hopkins Bloomberg School of Public Health, Baltimore, MD, USA. Verbal informed consent was obtained from all subjects. Verbal consent was witnessed and formally recorded. The maternal micronutrient supplementation cluster-randomised controlled trial, which provided the basis for the present study, was registered with ClinicalTrials.gov (NCT00860470).

\section{Results}

Out of 18288 identified births with complete data at maternal and SES assessment, 15051 (82.3\%) singletons were able to be assessed within 1 week $(<168 \mathrm{~h}$ ) after birth; this number resulted from 160 twins (0.9\%), 182 lost to follow-up (1.0\%) and 2895 measured beyond a 1 -week window (15.8\%). At 6 months postpartum, $927(5.1 \%)$ subjects were lost to follow-up. We further excluded subjects with missing data in the following: GA ( $n$ 567, 3.1\%), anthropometry measures at birth ( $n$ 319, $1.7 \%$ ) and at 6-month follow-up ( $n$ 439, $2.4 \%$ ), food security questions ( $n 2,<0 \cdot 1 \%$ ), feeding practice measures ( $n 99,0.5 \%$ ) and morbidity histories ( $n 5,<0 \cdot 1 \%$ ). Therefore, we kept $12693(69 \cdot 4 \%)$ mother-infant pairs in this analysis (Fig. 2): 6171 (48.6\%), 2600 (20.5\%), 1982 (15.6\%) and 1940 (15.3\%) households were categorised as food secure, mildly food insecure, moderately food insecure and severely food insecure, respectively.

Comparisons of women, infants and SES characteristics are demonstrated in Table 1 . On average, $73.7 \%$ and $24.8 \%$ of all mothers were measured within the first and second trimester of pregnancy, respectively, and these percentages did not differ by HFI group. Mothers in HFI groups tended to be older in age, to have more parity and to consume a less diverse diet than more food-secure women (all $P<0.001$ ). Infant age at 6 months follow-up $(P=0.37)$ and infant sex $(P=0.09)$ did not vary linearly by HFI status, although a lower proportion of female babies was observed among the severe HFI households. Risk of preterm birth rose monotonically from $16.9 \%$ in the foodsecure group to $20.9 \%$ in the severe HFI group $(P<0.001)$. While current breast-feeding at 6 months was universal $(100 \%)$, the reported frequency $(P=0.06)$ and sufficiency $(P<0 \cdot 001)$ of breast-feeding in the previous day decreased with increased HFI severity. The proportion of feeding formula, milk (powdered or fresh) or dairy food dropped linearly from foodsecure to food-insecure households (all $P<0 \cdot 001$ ). Feeding plain water was commonly practiced by $77.7 \%$ of all women on average and was not differentiable by HFI status $(P=0.32)$. Similar trends were seen in feeding semi-solid and solid foods: 


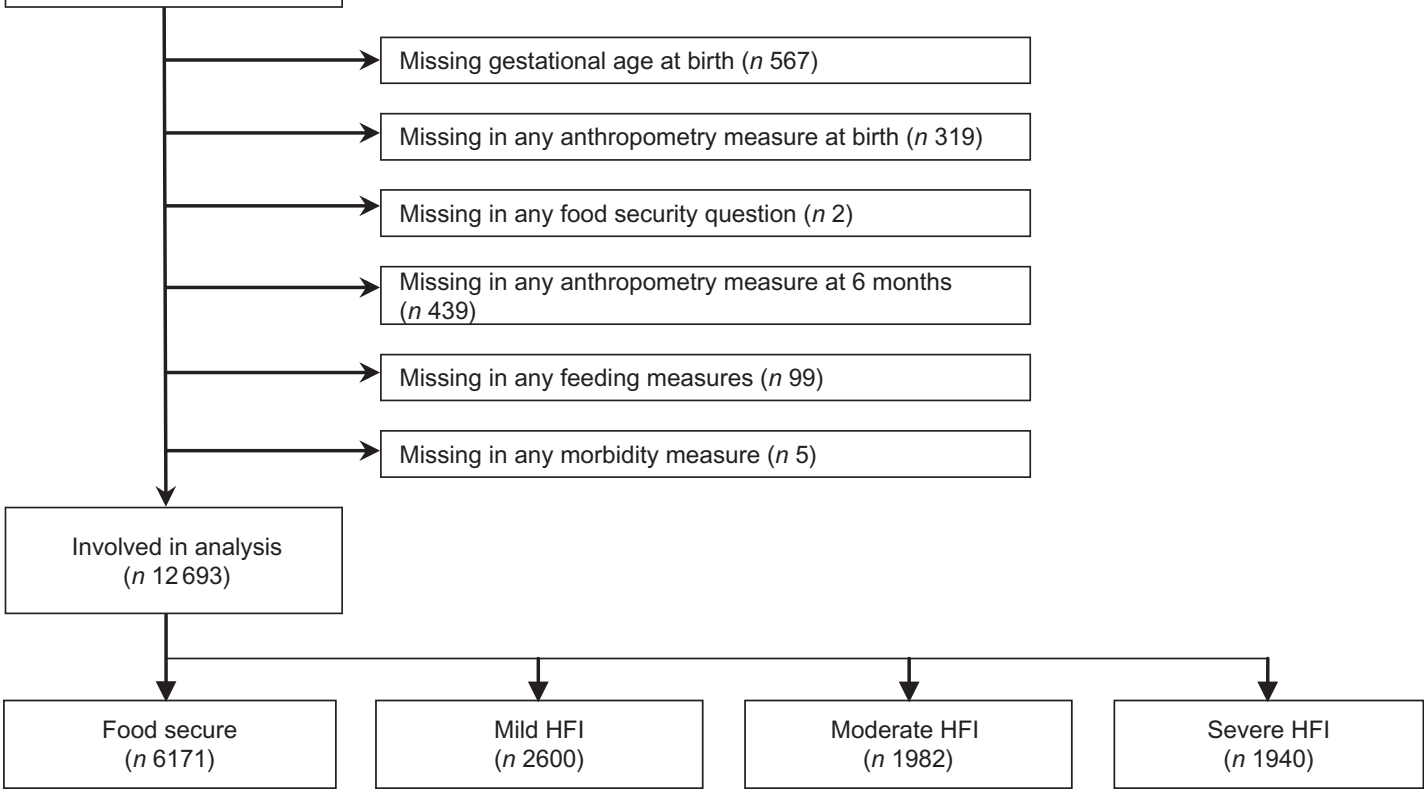

Fig. 2. Study population and food security categorisation. SES, socio-economic status; HFI, household food insecurity.

among commonly added food items such as foods made from grains $(62.2 \%, P=0.57)$ and biscuit $(55.0 \%, P=0.20)$, there was an equal distribution of such feeding practices across HFI groups. A general negative linear association was observed between increased HFI and a decrease in the proportion of feeding dal, banana, oil, sugar (all $P<0 \cdot 001$ ) and other foods $(P<0.05)$. Infants of food-insecure households tended to be more frequently ill for all four common infections in the previous $7 \mathrm{~d}$ than those from more food-secure households (all $P<0 \cdot 01$ ). SES variables were all significantly different by HFI status. Mothers progressively had less education as their HFI became more severe. However, the proportion of maternal employment differed across HFI groups in a non-linear way $(P<0.001)$ : $42.5 \%$ of mothers suffering severe HFI worked paid jobs, a proportion just slightly lower than the food-secure group (42.6\%) but higher than mothers from mild (39.9\%) and moderate
(37.6\%) households. Among food-secure households, 17.5 and $48.1 \%$ were in the lowest and highest wealth index tertile. The number gradually switched from 37.0 and $22.7 \%$, and 50.9 and $13.0 \%$, to 66.1 and $5.9 \%$ for the mild, moderate and severe HFI categories, respectively $(P<0.001)$. The season during which food insecurity was assessed showed heterogeneity in distribution by HFI groups $(P<0 \cdot 001)$. The maternal and infant anthropometric variables were normally distributed. As Table 2 shows, all anthropometric variables of women at early pregnancy, and of infants at birth and approximately 6 months of age, were negatively associated in a dose-responsive manner with increasing severity of food insecurity (all $P<0 \cdot 001$ ).

Mean infant size deficits comparing HFI groups against the food-secure reference group from the stepwise regression analyses are presented in Table 3. HFI shared a significant dose-responsive association with infant weight, length, BMI, 
Table 1. Characteristics of women, infants and household by household food security category ( $n 12693) \dagger$ (Numbers and percentages)

\begin{tabular}{|c|c|c|c|c|c|}
\hline & \multirow[b]{2}{*}{ Food secure $(n 6171)$} & \multicolumn{3}{|c|}{ Food insecurity } & \multirow[b]{2}{*}{$P \neq$} \\
\hline & & Mild (n 2600) & Moderate ( $n$ 1982) & Severe $(n 1940)$ & \\
\hline \multicolumn{6}{|l|}{ Mothers } \\
\hline \multicolumn{6}{|l|}{ Age (years) } \\
\hline$<20$ & $30 \cdot 4$ & 34.7 & 35.4 & $22 \cdot 6$ & 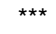 \\
\hline $20-29$ & $57 \cdot 0$ & $55 \cdot 5$ & $52 \cdot 3$ & $56 \cdot 9$ & \\
\hline$>29$ & $12 \cdot 7$ & $9 \cdot 8$ & $12 \cdot 3$ & 20.5 & \\
\hline \multicolumn{6}{|l|}{ Parity $(n)$} \\
\hline 0 & $36 \cdot 8$ & $36 \cdot 7$ & $36 \cdot 7$ & $22 \cdot 0$ & $* * *$ \\
\hline 1 & $37 \cdot 0$ & $36 \cdot 0$ & 31.5 & 28.5 & \\
\hline 2 & $17 \cdot 7$ & $18 \cdot 1$ & $18 \cdot 8$ & 24.4 & \\
\hline 3 & $5 \cdot 7$ & $6 \cdot 1$ & $8 \cdot 8$ & $14 \cdot 3$ & \\
\hline$\geq 4$ & $2 \cdot 8$ & $3 \cdot 1$ & $4 \cdot 1$ & $10 \cdot 8$ & \\
\hline \multicolumn{6}{|l|}{ WDDS tertiles } \\
\hline Low & 35.4 & $44 \cdot 0$ & $49 \cdot 3$ & $57 \cdot 7$ & *** \\
\hline Medium & $42 \cdot 4$ & $40 \cdot 3$ & 38.5 & $33 \cdot 6$ & \\
\hline High & $22 \cdot 2$ & $15 \cdot 8$ & $12 \cdot 2$ & $8 \cdot 7$ & \\
\hline \multicolumn{6}{|l|}{ Infants } \\
\hline \multicolumn{6}{|l|}{ Age at assessment (months) } \\
\hline$<6$ & 0.3 & 0.3 & 0.6 & 0.6 & 0.37 \\
\hline $6-7$ & $99 \cdot 1$ & $99 \cdot 1$ & $98 \cdot 8$ & $99 \cdot 0$ & \\
\hline$\geq 8$ & 0.6 & 0.6 & 0.6 & 0.5 & \\
\hline Female & $48 \cdot 7$ & $49 \cdot 8$ & 49.4 & $46 \cdot 2$ & 0.09 \\
\hline Preterm birth & $16 \cdot 9$ & $18 \cdot 9$ & $17 \cdot 7$ & $20 \cdot 9$ & $\star \star * *$ \\
\hline Current BF & $100 \cdot 0$ & $100 \cdot 0$ & $100 \cdot 0$ & $100 \cdot 0$ & \\
\hline \multicolumn{6}{|l|}{ BF frequency a day $(n)$} \\
\hline $1-10$ & $11 \cdot 2$ & 11.9 & $12 \cdot 6$ & $13 \cdot 7$ & 0.06 \\
\hline $11-20$ & 73.7 & $74 \cdot 2$ & $73 \cdot 8$ & $72 \cdot 3$ & \\
\hline$\geq 21$ & $15 \cdot 0$ & $13 \cdot 9$ & $13 \cdot 6$ & $14 \cdot 0$ & \\
\hline Had enough BF & $76 \cdot 2$ & 72.5 & $70 \cdot 6$ & $63 \cdot 3$ & *** \\
\hline \multicolumn{6}{|l|}{ Any food group given last week } \\
\hline Infant formula & $10 \cdot 7$ & 6.9 & $5 \cdot 0$ & 3.5 & *** \\
\hline Milk (powdered or fresh) & $24 \cdot 1$ & 19.5 & $16 \cdot 6$ & $16 \cdot 1$ & *** \\
\hline Dairy products & $6 \cdot 1$ & $5 \cdot 2$ & 3.9 & $2 \cdot 9$ & $\star \star * *$ \\
\hline Water & $77 \cdot 2$ & $78 \cdot 8$ & $78 \cdot 3$ & $77 \cdot 2$ & 0.32 \\
\hline Any grains & 61.6 & $62 \cdot 6$ & $62 \cdot 9$ & $63 \cdot 0$ & 0.57 \\
\hline Biscuit & 54.5 & $56 \cdot 0$ & $56 \cdot 6$ & 53.9 & 0.20 \\
\hline Dal & 4.8 & 4.5 & $2 \cdot 7$ & $2 \cdot 8$ & *** \\
\hline Banana & $11 \cdot 6$ & $10 \cdot 0$ & $10 \cdot 4$ & $8 \cdot 2$ & 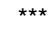 \\
\hline Added oil & 28.9 & $31 \cdot 0$ & $27 \cdot 9$ & 25.9 & 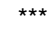 \\
\hline Added sugar & 33.9 & $33 \cdot 1$ & $29 \cdot 8$ & $29 \cdot 3$ & *** \\
\hline Other food & $24 \cdot 6$ & $24 \cdot 8$ & $23 \cdot 0$ & 21.4 & * \\
\hline \multicolumn{6}{|l|}{ Any symptom last week } \\
\hline Acute respiratory infections & $61 \cdot 3$ & 63.9 & 63.9 & $67 \cdot 4$ & 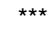 \\
\hline Diarrhoea & $2 \cdot 7$ & $3 \cdot 1$ & 4.4 & 4.6 & *** \\
\hline Bloody stools & 1.6 & 1.3 & 1.6 & $2 \cdot 7$ & ** \\
\hline Fever & $12 \cdot 9$ & 13.4 & $14 \cdot 0$ & $16 \cdot 3$ & ** \\
\hline \multicolumn{6}{|l|}{ Socio-economic variables } \\
\hline Maternal education (any schooling) & 83.8 & $76 \cdot 1$ & $66 \cdot 7$ & $52 \cdot 4$ & *** \\
\hline Maternal paid job & $42 \cdot 6$ & 39.9 & $37 \cdot 6$ & 42.5 & *** \\
\hline \multicolumn{6}{|l|}{ Wealth index tertiles } \\
\hline Low & 17.5 & $37 \cdot 0$ & $50 \cdot 9$ & $66 \cdot 1$ & *** \\
\hline Medium & 34.3 & $40 \cdot 3$ & $36 \cdot 2$ & 28.0 & \\
\hline High & $48 \cdot 1$ & $22 \cdot 7$ & $13 \cdot 0$ & 5.9 & \\
\hline \multicolumn{6}{|l|}{ Season of HFI assessment } \\
\hline Winter & $15 \cdot 1$ & $15 \cdot 6$ & 14.4 & $16 \cdot 5$ & *** \\
\hline Spring & $17 \cdot 2$ & $18 \cdot 1$ & $21 \cdot 2$ & $18 \cdot 4$ & \\
\hline Summer & $19 \cdot 3$ & 21.8 & $21 \cdot 2$ & $19 \cdot 7$ & \\
\hline Early monsoon & $19 \cdot 2$ & $16 \cdot 0$ & $14 \cdot 2$ & 13.4 & \\
\hline Later monsoon & $16 \cdot 5$ & $14 \cdot 3$ & 14.9 & $14 \cdot 6$ & \\
\hline Autumn & $12 \cdot 6$ & $14 \cdot 1$ & $14 \cdot 0$ & $17 \cdot 3$ & \\
\hline
\end{tabular}

WDDS, women's dietary diversity score; $\mathrm{BF}$, breast-feeding

${ }^{*} P<0.05,{ }^{* *} P<0.01,{ }^{* * *} P<0.001$.

† Sample size is the same for mothers and infants.

$\ddagger P$ value is from the $\chi^{2}$ test across HFI groups. 
Table 2. Women and infant anthropometry by household food insecurity category $\dagger$ (Mean values and standard deviations)

\begin{tabular}{|c|c|c|c|c|c|c|c|c|c|}
\hline & & & \multicolumn{6}{|c|}{ Food insecurity } & \multirow[b]{3}{*}{$P$} \\
\hline & \multicolumn{2}{|c|}{ Food secure } & \multicolumn{2}{|c|}{ Mild } & \multicolumn{2}{|c|}{ Moderate } & \multicolumn{2}{|c|}{ Severe } & \\
\hline & Mean & SD & Mean & SD & Mean & SD & Mean & SD & \\
\hline \multicolumn{10}{|l|}{ Mothers } \\
\hline Weight (kg) & 44.2 & 6.5 & $43 \cdot 2$ & $6 \cdot 0$ & 42.5 & $5 \cdot 7$ & $42 \cdot 0$ & 5.5 & $\star \star \star \star$ \\
\hline Height (cm) & $150 \cdot 2$ & $5 \cdot 1$ & 149.6 & $5 \cdot 2$ & $149 \cdot 2$ & $5 \cdot 0$ & 148.8 & 5.4 & $\star \star \star \star ~$ \\
\hline MUAC $(\mathrm{cm})$ & 23.8 & $2 \cdot 3$ & 23.5 & $2 \cdot 2$ & 23.3 & $2 \cdot 1$ & 23.0 & $2 \cdot 0$ & $\star \star \star *$ \\
\hline $\mathrm{BMI}\left(\mathrm{kg} / \mathrm{m}^{2}\right)$ & 19.5 & 2.5 & $19 \cdot 3$ & $2 \cdot 3$ & $19 \cdot 1$ & $2 \cdot 2$ & $18 \cdot 9$ & $2 \cdot 1$ & *** \\
\hline \multicolumn{10}{|l|}{ Infant at birth } \\
\hline Weight (g) & 2612 & 401 & 2571 & 400 & 2550 & 396 & 2555 & 406 & $\star * *$ \\
\hline Height (cm) & 46.8 & $2 \cdot 1$ & $46 \cdot 6$ & $2 \cdot 2$ & $46 \cdot 6$ & $2 \cdot 2$ & $46 \cdot 6$ & $2 \cdot 2$ & $\star \star \star *$ \\
\hline MUAC $(\mathrm{cm})$ & $9 \cdot 6$ & 0.8 & 9.5 & 0.8 & 9.5 & 0.8 & 9.5 & 0.8 & *** \\
\hline $\mathrm{HC}(\mathrm{cm})$ & $32 \cdot 8$ & 1.5 & $32 \cdot 6$ & 1.5 & 32.6 & 1.5 & $32 \cdot 6$ & 1.6 & *** \\
\hline $\mathrm{CC}(\mathrm{cm})$ & 31.0 & $2 \cdot 0$ & $30 \cdot 8$ & 2.0 & 30.8 & 2.0 & 30.7 & 2.0 & $* * *$ \\
\hline $\mathrm{PI}\left(\mathrm{kg} / \mathrm{m}^{3}\right)$ & $25 \cdot 3$ & $2 \cdot 4$ & $25 \cdot 2$ & $2 \cdot 4$ & $25 \cdot 1$ & $2 \cdot 4$ & $25 \cdot 1$ & 2.5 & *** \\
\hline \multicolumn{10}{|c|}{ Infants at 6-month visit } \\
\hline Weight (g) & 6732 & 849 & 6639 & 819 & 6602 & 848 & 6548 & 862 & $\star \star \star \star$ \\
\hline Height $(\mathrm{cm})$ & 64.4 & 2.5 & $64 \cdot 1$ & $2 \cdot 4$ & $64 \cdot 0$ & 2.5 & $64 \cdot 0$ & $2 \cdot 6$ & *** \\
\hline MUAC $(\mathrm{cm})$ & $13 \cdot 3$ & 1.0 & 13.3 & 1.0 & 13.2 & 1.0 & $13 \cdot 1$ & 1.0 & $* * *$ \\
\hline $\mathrm{HC}(\mathrm{cm})$ & 41.6 & 1.4 & 41.5 & 1.3 & 41.5 & 1.4 & 41.4 & 1.4 & $\star * *$ \\
\hline $\mathrm{CC}(\mathrm{cm})$ & 42.4 & $2 \cdot 1$ & $42 \cdot 2$ & $2 \cdot 0$ & $42 \cdot 1$ & $2 \cdot 1$ & $42 \cdot 0$ & $2 \cdot 2$ & $* * *$ \\
\hline BMI $\left(\mathrm{kg} / \mathrm{m}^{2}\right)$ & $16 \cdot 2$ & 1.5 & $16 \cdot 1$ & 1.5 & $16 \cdot 1$ & 1.5 & $16 \cdot 0$ & 1.5 & $* * *$ \\
\hline
\end{tabular}

MUAC, mid-upper arm circumference; $\mathrm{HC}$, head circumference; CC, chest circumference; PI, ponderal index, calculated as weight $(\mathrm{kg}) /\left(\right.$ length $\left.(\mathrm{m})^{3}\right)$.

*** $P<0.001$.

† Sample size is the same for mothers and infants.

$\ddagger P$ value is from the non-parametric test for linear trend across $\mathrm{HFI}$ groups.

and MUAC, head circumference, and chest circumference at 6 months of age. Compared with the reference, mean differences in child sizes were negative and the deficits enlarged progressively from the mild through the moderate to severe HFI group. Such dose-responsive relationships held true in the unadjusted models and along the cumulatively adjusted models in general. In all three HFI groups, the deficits in child sizes decreased with the sequential adjustments, except after adjusting for non-breast milk feeding practices, in which the size deficits became slightly greater. Compared with model 0 , the decreasing trend in size deficits occurred largely after adjusting for prenatal maternal nutritional factors (model 2), and birth size (model 3), by 39-67 and 11-33\%, respectively. Together maternal nutrition and birth sizes explained $57-89 \%$ of the size deficits found in unadjusted models. Postnatal factors, such as feeding practices and child morbidity altogether, further brought down the mean size differences by another $0-17 \%$ (model 4 ). Other contextual variables, including maternal employment, maternal education, wealth index and seasonality, explained $0-36 \%$ of the remaining differences in infant sizes (model 5). For infant weight, length and BMI, almost all size differences lost statistical significance after maternal nutrition was adjusted (model 2). For infant MUAC, head circumference and chest circumference, deficits between HFI groups were statistically insignificant and practically zero after birth size measures were further included (model 3).

Table 4 shows the estimated relative risk of mid-infancy malnutrition with similar sequential adjustments. Risk of wasting in the mild or moderate group was not different at any level of adjustment compared with infants from food-secure families.
Severe HFI was associated with a $36 \%$ (95\% CI 14\%, 61\%) increased odds of being wasted, which decreased to $17 \%$ (95\% CI $-3 \%, 40 \%$ ) after adjustment for maternal nutrition and remained insignificant thereafter. The dose-responsive relationship was observed between HFI and risk of infant stunting and underweight. Compared with the food-secure group in model 0 , mild, moderate and severe HFI were associated with a 6, 27 and 39\% increased risk for stunting and an 18, 37 and $62 \%$ increased risk for underweight, respectively. Relative to food-secure infants after adjusting for maternal height and MUAC, the increased odds of stunting and underweight in ascending order of HFI categories dropped to $-3,10$ and $16 \%$, and to 8,19 and $32 \%$, respectively, about halved from their unadjusted level (model 0). After model 3 with adjustment of birth size, risk of stunting and underweight decreased by another $5.8 \%$ on average for all HFI groups to an insignificant level. Exceptionally, severe HFI was still significantly associated with a $25 \%$ (95\% CI 9\%, 42\%) increased risk of underweight at this point, which went down to $17 \%$ (95\% CI 2\%, 34\%) when accounting for postnatal feeding practices and morbidity variables (model 4) and then to an insignificant level of $7 \%$ in the full model with contextual variables (model 5). Compared with the food-secure group, mild HFI was now associated with a $13 \%$ (95\% CI 1\%, 24\%) lower risk of mid-infancy stunting in the full model.

\section{Discussion}

Within this typical rural setting of northern Bangladesh, we sought to explain the relationship between HFI, assessed by a 


\section{Writish Journal of Nutrition}

Table 3. Mean differences in infant size at 6 months between infants from food-insecure households and infants from food-secure households (reference group)

(Mean values with their standard errors)

\begin{tabular}{|c|c|c|c|c|c|c|c|c|c|c|c|c|c|c|c|c|c|c|}
\hline & \multicolumn{6}{|c|}{ Weight (g) } & \multicolumn{6}{|c|}{ Length $(\mathrm{cm})$} & \multicolumn{6}{|c|}{$\mathrm{BMI}\left(\mathrm{kg} / \mathrm{m}^{2}\right)$} \\
\hline & \multicolumn{2}{|c|}{ Mild } & \multicolumn{2}{|c|}{ Moderate } & \multicolumn{2}{|c|}{ Severe } & \multicolumn{2}{|c|}{ Mild } & \multicolumn{2}{|c|}{ Moderate } & \multicolumn{2}{|c|}{ Severe } & \multicolumn{2}{|c|}{ Mild } & \multicolumn{2}{|c|}{ Moderate } & \multicolumn{2}{|c|}{ Severe } \\
\hline & Mean & SE & Mean & SE & Mean & SE & Mean & SE & Mean & SE & Mean & SE & Mean & SE & Mean & $\mathrm{SE}$ & Mean & $\mathrm{SE}$ \\
\hline \multicolumn{19}{|l|}{$\begin{array}{l}\text { Model 0: crude estimates } \\
\text { Unadiusted }\end{array}$} \\
\hline \\
\hline Infant sex + age & $-86^{\star}$ & 18 & $-125^{\star}$ & 20 & $-196^{\star}$ & 21 & $-0.26^{*}$ & 0.05 & $-0.39^{*}$ & 0.06 & $-0.48^{\star}$ & 0.06 & $-0.08^{*}$ & 0.03 & $-0.11^{*}$ & 0.04 & $-0.25^{*}$ & 0.04 \\
\hline \multicolumn{19}{|l|}{$\begin{array}{l}\text { Parity + maternal age }+ \text { maternal GA } \\
\text { Model 2: additionally adjusted for prenatal factors }\end{array}$} \\
\hline Maternal height & $-58^{*}$ & 18 & $-78^{*}$ & 20 & $-133^{*}$ & 20 & $-0.15^{*}$ & 0.05 & $-0.21^{*}$ & 0.06 & $-0.28^{*}$ & 0.06 & -0.06 & 0.03 & $-0.09^{*}$ & 0.04 & $-0.19^{*}$ & 0.04 \\
\hline Maternal MUAC & $-44^{*}$ & 18 & $-54^{*}$ & 20 & $-94^{*}$ & 20 & $-0.13^{*}$ & 0.05 & $-0.18^{*}$ & 0.06 & $-0.23^{*}$ & 0.06 & -0.04 & 0.03 & -0.05 & 0.04 & $-0.12^{*}$ & 0.04 \\
\hline \multirow{2}{*}{\multicolumn{19}{|c|}{ Model 3: additionally adjusted for birth size measures }} \\
\hline & & & & & & & & & & & & & & & & & & \\
\hline $\begin{array}{l}\text { Infant's GA at birth } \\
\text { Bith lengtht }\end{array}$ & $-37^{\star}$ & 18 & $-47^{*}$ & 20 & $-81^{*}$ & 20 & $-0.10^{*}$ & 0.05 & $-0.15^{*}$ & 0.06 & $-0.18^{*}$ & 0.06 & -0.03 & 0.03 & -0.04 & 0.04 & $-0.12^{*}$ & 0.04 \\
\hline Birth length $\uparrow$ & -29 & 16 & -31 & 18 & $-60^{*}$ & 18 & -0.07 & 0.04 & $-0.09^{*}$ & 0.05 & $-0.1^{\star}$ & 0.05 & -0.03 & 0.03 & -0.03 & 0.04 & $-0.11^{*}$ & 0.04 \\
\hline Ponderal index $\neq$ & -21 & 15 & -16 & 17 & $-45^{\star}$ & 18 & -0.07 & 0.04 & -0.09 & 0.05 & -0.09 & 0.05 & -0.01 & 0.03 & 0 & 0.04 & -0.07 & 0.04 \\
\hline \multicolumn{19}{|l|}{ Model 4: additionally adjusted for postnatal factors } \\
\hline BF practices $\S$ & -14 & 15 & -6 & 17 & -22 & 18 & -0.06 & 0.04 & -0.08 & 0.05 & -0.07 & 0.05 & 0 & 0.03 & 0.02 & 0.04 & -0.03 & 0.04 \\
\hline CF practices $\|$ & -16 & 15 & -9 & 17 & -25 & 18 & -0.06 & 0.04 & -0.07 & 0.05 & -0.07 & 0.05 & 0 & 0.03 & 0.01 & 0.04 & -0.04 & 0.04 \\
\hline Child morbidityq & -16 & 15 & -7 & 17 & -21 & 18 & -0.06 & 0.04 & -0.07 & 0.05 & -0.06 & 0.05 & 0 & 0.03 & 0.01 & 0.04 & -0.03 & 0.04 \\
\hline \multicolumn{19}{|l|}{ Model 5: additionally adjusted for contextual factors } \\
\hline Maternal employment* ${ }^{\star \star}$ & -16 & 15 & -7 & 17 & -21 & 18 & -0.06 & 0.04 & -0.07 & 0.05 & -0.07 & 0.05 & 0 & 0.03 & 0.01 & 0.04 & -0.03 & 0.04 \\
\hline Maternal education†† & -11 & 15 & 0 & 17 & -12 & 18 & -0.05 & 0.04 & -0.05 & 0.05 & -0.04 & 0.05 & 0 & 0.03 & 0.02 & 0.04 & -0.02 & 0.04 \\
\hline Wealth index & 1 & 16 & 17 & 18 & 8 & 19 & -0.03 & 0.04 & -0.03 & 0.05 & -0.01 & 0.05 & 0.02 & 0.03 & 0.05 & 0.04 & 0.01 & 0.04 \\
\hline Season & 2 & 16 & 18 & 18 & 8 & 19 & -0.02 & 0.04 & -0.01 & 0.05 & 0.01 & 0.05 & 0.02 & 0.03 & 0.04 & 0.04 & 0 & 0.04 \\
\hline & & & MUAC & & & & & & $\mathrm{HC}$ & & & & & & $\mathrm{CC}$ & & & \\
\hline & M & & Mode & & Sev & & & & Mod & & & & $\mathrm{Mi}$ & & Mod & & Sev & \\
\hline & Mean & $\mathrm{SE}$ & Mean & SE & Mean & $\mathrm{SE}$ & Mean & SE & Mean & SE & Mean & SE & Mean & SE & Mean & SE & Mean & SE \\
\hline Model 0: crude estimates & & & & & & & & & & & & & & & & & & \\
\hline $\begin{array}{l}\text { Unadjusted } \\
\text { Model 1: adjusted for infant and maternal factors }\end{array}$ & $-0.08^{*}$ & 0.02 & $-0.14^{\star}$ & 0.03 & $-0.21^{*}$ & 0.03 & $-0.08^{*}$ & 0.03 & $-0.14^{*}$ & 0.04 & $-0.19^{*}$ & 0.04 & $-0.17^{*}$ & 0.05 & $-0.31^{*}$ & 0.05 & $-0.41^{*}$ & 0.06 \\
\hline 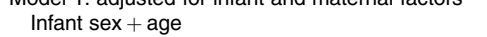 & $-0.08^{*}$ & 0.02 & $-0.13^{*}$ & 0.02 & $-0.22^{*}$ & 0.02 & $-0.06^{*}$ & 0.03 & $-0.13^{*}$ & 0.03 & $-0.22^{*}$ & 0.03 & $-0.16^{*}$ & 0.05 & $-0.30^{*}$ & 0.05 & $-0.43^{*}$ & 0.05 \\
\hline $\begin{array}{l}\text { Parity + maternal age + maternal GA } \\
\text { Model 2. additionally adiusted for prenatal factors }\end{array}$ & $-0.07^{*}$ & 0.02 & $-0.12^{*}$ & 0.02 & $-0.19^{*}$ & 0.03 & -0.05 & 0.03 & $-0.12^{*}$ & 0.03 & $-0.21^{*}$ & 0.03 & $-0.14^{*}$ & 0.05 & $-0.28^{*}$ & 0.05 & $-0.40^{*}$ & 0.05 \\
\hline Maternal height & $-0.06^{*}$ & 0.02 & $-0.10^{*}$ & 0.02 & $-0.16^{*}$ & 0.03 & -0.03 & 0.03 & $-0.07^{*}$ & 0.03 & $-0.15^{*}$ & 0.03 & $-0.10^{*}$ & 0.05 & $-0.20^{*}$ & 0.05 & $-0.29^{*}$ & 0.05 \\
\hline Maternal MUAC & -0.04 & 0.02 & $-0.06^{*}$ & $0 . c$ & $-0.11^{*}$ & 0.0 & -0.01 & 0.03 & -0.05 & 0.03 & $-0.11^{*}$ & 0.03 & -0.07 & 0.05 & $-0.15^{*}$ & 0.05 & $-0.21^{*}$ & 0.05 \\
\hline WDDS & -0.03 & 0.02 & $-0.06^{*}$ & 0.02 & $-0.10^{*}$ & 0.03 & 0 & 0.03 & -0.03 & 0.03 & $-0.09^{*}$ & 0.03 & -0.06 & 0.05 & $-0.14^{\star}$ & 0.05 & $-0.19^{*}$ & 0.05 \\
\hline Model 3: additionally adjusted for birth size measur & & & & & & & & & & & & & & & & & & \\
\hline $\begin{array}{l}\text { Infant's GA at birth } \\
\text { Bith lengtht }\end{array}$ & -0.03 & 0.02 & $-0.06^{*}$ & 0.02 & $-0.10^{*}$ & 0.03 & 0 & 0.03 & -0.03 & 0.03 & $-0.09^{*}$ & 0.03 & -0.06 & 0.05 & $-0.14^{*}$ & 0.05 & $-0.18^{*}$ & 0.05 \\
\hline $\begin{array}{l}\text { Birth length } \dagger \\
\text { Ponderal index } \neq\end{array}$ & $\begin{array}{l}-0.03 \\
-0.02\end{array}$ & $\begin{array}{l}0.02 \\
0.02\end{array}$ & $\begin{array}{l}-0.05 \\
-0.03\end{array}$ & $\begin{array}{l}0.02 \\
0.02\end{array}$ & $\begin{array}{l}-0.08^{*} \\
-0.06^{*}\end{array}$ & $\begin{array}{l}0.02 \\
0.02\end{array}$ & $\begin{array}{l}0.01 \\
0.02\end{array}$ & 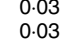 & $\begin{array}{l}-0.01 \\
0\end{array}$ & $\begin{array}{l}0.03 \\
0.03\end{array}$ & $\begin{array}{l}-0.00^{*} \\
-0.04\end{array}$ & 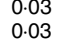 & $\begin{array}{l}-0.04 \\
-0.02\end{array}$ & $\begin{array}{l}0.04 \\
0.04\end{array}$ & $\begin{array}{l}-0.11^{*} \\
-0.07\end{array}$ & $\begin{array}{l}0.05 \\
0.05\end{array}$ & $\begin{array}{l}-0.11^{*} \\
-0.11^{*}\end{array}$ & $\begin{array}{l}0.05 \\
0.05\end{array}$ \\
\hline Model 4: additionally adjusted for postnatal factors & & & & & & & & & & & & & & & & & & \\
\hline BF practices§ & -0.01 & 0.02 & -0.02 & 0.02 & -0.04 & 0.02 & 0.03 & 0.03 & 0.01 & 0.03 & -0.03 & 0.03 & -0.01 & 0.04 & -0.05 & 0.05 & -0.06 & 0.05 \\
\hline CF practices\| & -0.01 & 0.02 & -0.02 & 0.02 & -0.04 & 0.02 & 0.02 & 0.03 & 0.01 & 0.03 & -0.02 & 0.0 & -0.01 & 0.04 & -0.06 & 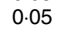 & -0.07 & 0.05 \\
\hline $\begin{array}{l}\text { Child morbidityfl } \\
\text { and }\end{array}$ & -0.01 & 0.02 & -0.02 & 0.02 & -0.04 & 0.02 & 0.02 & 0.03 & 0.01 & 0.03 & -0.02 & 0.03 & -0.01 & 0.04 & -0.05 & 0.05 & -0.06 & 0.05 \\
\hline Model 5: additionally adjusted for contextual factors & & & & & & & & & & & & & & & & & & \\
\hline $\begin{array}{l}\text { Maternal employment** } \\
\text { Maternal educationtt }\end{array}$ & $\begin{array}{c}-0.01 \\
0\end{array}$ & $\begin{array}{l}0.02 \\
0.02\end{array}$ & $\begin{array}{c}-0.02 \\
0\end{array}$ & $\begin{array}{l}0.02 \\
0.02\end{array}$ & $\begin{array}{l}-0.03 \\
-0.01\end{array}$ & $\begin{array}{l}0.02 \\
0.02\end{array}$ & $\begin{array}{l}0.03 \\
0.03\end{array}$ & $\begin{array}{l}0.03 \\
0.03\end{array}$ & 0.02 & 0.03 & $\begin{array}{l}-0.02 \\
-0.02\end{array}$ & 0.03 & -0.01 & 0.04 & -0.05 & 0.05 & -0.05 & $\begin{array}{l}0.05 \\
0.05\end{array}$ \\
\hline $\begin{array}{l}\text { Maternal education } \dagger \\
\text { Wealth index }\end{array}$ & 0.02 & $\begin{array}{l}0.02 \\
0.02\end{array}$ & 0.02 & 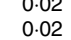 & 0.01 & $\begin{array}{l}0.02 \\
0.03\end{array}$ & $\begin{array}{l}0.03 \\
0.04\end{array}$ & $\begin{array}{l}0.03 \\
0.03\end{array}$ & $\begin{array}{l}0.02 \\
0.03\end{array}$ & $\begin{array}{l}0.03 \\
0.03\end{array}$ & $\begin{array}{c}-0.02 \\
0\end{array}$ & $\begin{array}{l}0.03 \\
0.03\end{array}$ & $\begin{array}{l}0 \\
0.04\end{array}$ & $\begin{array}{l}0.04 \\
0.04\end{array}$ & $\begin{array}{r}-0.03 \\
0.02\end{array}$ & $\begin{array}{l}0.05 \\
0.05\end{array}$ & $\begin{array}{r}-0.03 \\
0.04\end{array}$ & $\begin{array}{l}0.05 \\
0.05\end{array}$ \\
\hline Season & 0.02 & 0.02 & 0.03 & 0.02 & 0.02 & 0.03 & 0.04 & 0.03 & 0.03 & 0.03 & 0 & 0.03 & 0.04 & 0.04 & 0.02 & 0.05 & 0.03 & 0.05 \\
\hline
\end{tabular}

GA, gestational age; MUAC, mid-upper arm circumference; WDDS, women's dietary diversity score; BF, breast-feeding; CF, complementary feeding; HC, head circumference; CC, chest circumference.

Significant at 0.05 level.

† Birth length is adjusted for the hour interval of measurement since delivery.

§ Ponderal index is calculated as birth weight $(\mathrm{kg}) /$ length $(\mathrm{m})^{3}$.

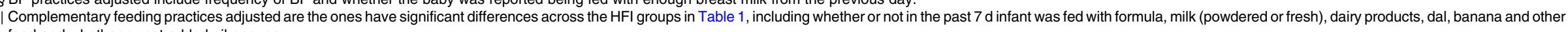
food and whether or not added oil or sugar.

II Child morbidity adjusted includes whether or not infant had morbidity symptoms of acute respiratory, diarrhoea, dysentery and fever in the previous $7 \mathrm{~d}$

* Maternal employment is whether or not mother had a paid job at enrolment. 
Table 4. Infant malnutrition at 6 months of age in infants from food-insecure households as compared with infants from food-secure households (reference group) (Odds ratios and $95 \%$ confidence intervals)

\begin{tabular}{|c|c|c|c|c|c|c|c|c|c|c|c|c|c|c|c|c|c|c|}
\hline & \multicolumn{6}{|c|}{ Wasting (WLZ <-2) } & \multicolumn{6}{|c|}{ Stunting $(L A Z<-2)$} & \multicolumn{6}{|c|}{ Underweight (WAZ <-2) } \\
\hline & \multicolumn{2}{|r|}{ Mild } & \multicolumn{2}{|c|}{ Moderate } & \multicolumn{2}{|c|}{ Severe } & \multicolumn{2}{|r|}{ Mild } & \multicolumn{2}{|c|}{ Moderate } & \multicolumn{2}{|c|}{ Severe } & \multicolumn{2}{|r|}{ Mild } & \multicolumn{2}{|c|}{ Moderate } & \multicolumn{2}{|c|}{ Severe } \\
\hline & OR & $95 \% \mathrm{Cl}$ & OR & $95 \% \mathrm{Cl}$ & OR & $95 \% \mathrm{Cl}$ & OR & $95 \% \mathrm{Cl}$ & OR & $95 \% \mathrm{Cl}$ & OR & $95 \% \mathrm{Cl}$ & OR & $95 \% \mathrm{Cl}$ & OR & $95 \% \mathrm{Cl}$ & OR & $95 \% \mathrm{Cl}$ \\
\hline \multicolumn{19}{|c|}{$\begin{array}{lllllllllll}1.24 & 1.55 & 1.18^{*} & 1.06 & 1.31 & 1.37^{*} & 1.22 & 1.54 & 1.62^{*} & 1.44 & 1.81\end{array}$} \\
\hline Unadjusted & $1 \cdot 13$ & $0.96,1.33$ & $1 \cdot 11$ & $0.92,1.33$ & $1.36^{*}$ & $1.14,1.61$ & 1.06 & $0.96,1 \cdot 18$ & $1 \cdot 27^{\star}$ & $1.13,1.42$ & $1.39^{*}$ & $1.24,1.55$ & $1 \cdot 18^{*}$ & $1 \cdot 06,1.31$ & $1 \cdot 37^{*}$ & $1.22,1.54$ & $1.62^{\star}$ & $1.44,1.81$ \\
\hline Model 1: adjusted for infant and maternal factors & & & & & & & & & & & & & & & & & & \\
\hline $\begin{array}{l}\text { Parity + maternal age + maternal GA } \\
\text { Model 2: additionally adjusted for prenatal factors }\end{array}$ & 1.12 & $0.95,1.33$ & 1.09 & $0.91,1.31$ & $1 \cdot 30^{*}$ & $1.09,1.55$ & 1.05 & $0.94,1 \cdot 16$ & $1 \cdot 24^{*}$ & $1.11,1.39$ & $1.39^{*}$ & $1.24,1.56$ & $1 \cdot 16^{*}$ & $1.04,1 \cdot 29$ & $1.34^{*}$ & $1.20,1.51$ & $1.58^{\star}$ & $1.41,1.77$ \\
\hline Maternal height & $1 \cdot 11$ & $0.94,1.32$ & 1.08 & $0.90,1.29$ & $1 \cdot 27^{\star}$ & $1.06,1.52$ & 0.98 & $0.88,1.09$ & $1 \cdot 12$ & $1.00,1.26$ & $1 \cdot 20^{*}$ & $1.07,1.36$ & $1 \cdot 11$ & $0.99,1.24$ & $1 \cdot 25^{*}$ & $1.11,1.40$ & $1.42^{*}$ & $1.26,1.60$ \\
\hline Maternal MUAC & 1.08 & $0.92,1.28$ & 1.02 & $0.85,1.23$ & 1.17 & $0.97,1.40$ & 0.97 & $0.86,1.08$ & $1 \cdot 10$ & $0.97,1.23$ & $1 \cdot 16^{*}$ & $1.03,1.31$ & 1.08 & $0.97,1.21$ & $1 \cdot 19^{*}$ & $1.06,1.34$ & $1.32^{\star}$ & $1.17,1.49$ \\
\hline WDDS & 1.08 & $0.91,1.27$ & 1.01 & $0.84,1.22$ & 1.15 & $0.96,1.38$ & 0.96 & $0.86,1.07$ & 1.08 & $0.96,1.22$ & $1 \cdot 14^{\star}$ & $1.00,1.28$ & 1.07 & $0.96,1 \cdot 20$ & $1 \cdot 17^{*}$ & $1.04,1.32$ & $1.29^{\star}$ & $1.14,1.46$ \\
\hline \multicolumn{19}{|c|}{ Model 3: additionally adjusted for birth size measures } \\
\hline Infant's GA at birth & 1.08 & $0.91,1.27$ & 1.01 & $0.84,1.22$ & $1 \cdot 15$ & $0.96,1.39$ & 0.95 & $0.85,1.06$ & 1.08 & & 1.12 & & 1.06 & $0.95,1.19$ & $1 \cdot 17^{*}$ & $1.04,1.32$ & $1 \cdot 28^{\star}$ & $1.13,1.45$ \\
\hline Birth I & 1.07 & $0.91,1.27$ & 1.00 & $0.83,1.21$ & 1.15 & $0.95,1.38$ & 0.92 & $0.81,1.04$ & 1.03 & $0.90,1 \cdot 18$ & 1.07 & $0.93,1.23$ & 1.06 & $0.94,1 \cdot 19$ & $1 \cdot 14^{*}$ & $1.01,1.30$ & $1.26^{*}$ & $1.11,1.44$ \\
\hline Ponderal index $\ddagger$ & 1.05 & $0.88,1.24$ & 0.96 & $0.79,1 \cdot 16$ & 1.09 & $0.91,1.32$ & 0.92 & $0.81,1.04$ & 1.02 & $0.90,1.17$ & 1.06 & $0.93,1.22$ & 1.05 & $0.93,1.18$ & 1.11 & $0.98,1.27$ & $1 \cdot 22^{*}$ & $1.07,1.40$ \\
\hline \multicolumn{19}{|c|}{ Model 4: additionally adjusted for postnatal factors } \\
\hline BF practices§ & 1.03 & $0.87,1.22$ & 0.93 & $0.77,1.12$ & 1.02 & $0.85,1.24$ & 0.91 & $0.80,1.03$ & 1.01 & $0.88,1 \cdot 16$ & 1.03 & $0.90,1.18$ & 1.02 & $0.91,1.16$ & 1.08 & $0.95,1.23$ & $1 \cdot 15^{\star}$ & $1.00,1.31$ \\
\hline CF practices & 1.04 & $0.88,1.24$ & 0.94 & $0.78,1.14$ & 1.05 & $0.87,1.26$ & 0.91 & $0.80,1.03$ & 1.02 & $0.89,1 \cdot 17$ & 1.05 & $0.91,1.20$ & 1.03 & $0.91,1 \cdot 17$ & $1 \cdot 10$ & $0.96,1.25$ & $1 \cdot 17^{\star}$ & $1.02,1.33$ \\
\hline Child morbidity & 1.04 & $0.88,1.23$ & 0.94 & $0.78,1.14$ & 1.03 & $0.86,1.25$ & 0.91 & $0.80,1.03$ & 1.02 & $0.89,1.17$ & 1.04 & $0.90,1.20$ & 1.03 & $0.91,1 \cdot 16$ & 1.09 & $0.95,1.24$ & $1 \cdot 15^{\star}$ & $1.00,1.32$ \\
\hline \multicolumn{19}{|c|}{ Model 5: additionally adjusted for contextual factors } \\
\hline Maternal employment* ${ }^{*}$ & 1.04 & $0.88,1.23$ & 0.94 & $0.77,1.13$ & 1.03 & $0.85,1.24$ & 0.91 & $0.80,1.03$ & 1.02 & $0.89,1.17$ & 1.04 & & 1.03 & & 1.08 & & $1 \cdot 15^{\star}$ & $1.00,1.31$ \\
\hline I educationtt & 1.04 & $0.87,1.23$ & 0.93 & $0.77,1.13$ & 1.02 & $0.84,1.24$ & 0.90 & $0.79,1.02$ & 0.99 & $0.86,1 \cdot 14$ & 1.01 & $0.87,1$. & 1.02 & $0,1 \cdot 15$ & 1.06 & $3,1.22$ & $1 \cdot 12$ & $0.97,1.28$ \\
\hline Wealth inde & 1.00 & $0.84,1 \cdot 19$ & 0.88 & $0.73,1.08$ & 0.96 & $0.78,1 \cdot 17$ & $0.87^{\star}$ & $0.77,0.99$ & 0.96 & $0.83,1.11$ & 0.97 & $0.83,1.12$ & 0.99 & $0.87,1.12$ & 1.02 & $0.89,1.18$ & 1.06 & $0.92,1.23$ \\
\hline Season & 0.99 & $0.83,1 \cdot 18$ & 0.88 & $0.72,1.07$ & 0.95 & $0.78,1 \cdot 16$ & $0.87^{\star}$ & $0.76,0.99$ & 0.95 & $0.82,1 \cdot 10$ & 0.96 & $0.82,1.11$ & 0.99 & $0.87,1.12$ & 1.02 & $0.88,1.17$ & 1.07 & $0.92,1.23$ \\
\hline
\end{tabular}

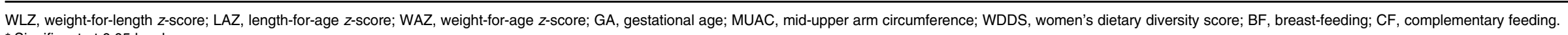
* Significant at 0.05 level.

† Birth length is adjusted for the hour interval of measurement since delivery.

† Ponderal index is calculated as birth weight $(\mathrm{kg}) /$ length $(\mathrm{m})^{3}$.

$\S \mathrm{BF}$ practices adjusted include frequency of $\mathrm{BF}$ and whether the baby was reported being fed with enough breast milk from the previous day.

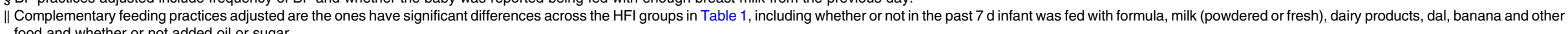
food and whether or not added oil or sugar.

I Child morbidity adjusted includes whether or not infant had morbidity symptoms of acute respiratory, diarrhoea, dysentery and fever in the previous $7 \mathrm{~d}$.

tt Maternal education is the highest women have completed. 
nine-item questionnaire, and mid-infancy anthropometric indicators of wasting and stunting malnutrition. We reasoned that HFI, if sufficiently severe and extended into pregnancy, may significantly affect attained postnatal growth. Further, we sought to identify determinants of any observed association by introducing potentially causal, antecedent indicators of prenatal maternal nutritional status, newborn GA and size reflecting health and nutrition during gestation, postnatal dietary and morbidity exposures and contextual household SES conditions that could explain the association between HFI and mid-infancy status. A substantial proportion of growth deficits observed by HFI status can be explained by maternal nutritional status at early pregnancy and nutrition during pregnancy (assessed by birth size), suggesting a sensitive intervention period to address HFI-related child malnutrition starting in and even before pregnancy in food-insecure mothers.

Our findings revealed a consistent, dose-response decline in attained infant ponderal and linear growth at 6 months of age with increasing severity of a home food insecurity index. Infants from households classified as severely food insecure were $184 \mathrm{~g}$ lighter, $0.45 \mathrm{~cm}$ shorter, $0.2 \mathrm{~cm}$ less in arm and head circumferences, $0.4 \mathrm{~cm}$ less in chest circumference and $0.23 \mathrm{~kg} / \mathrm{m}^{2}$ less than infants in food-secure homes. Further, each categorical decrement in HFI from adequacy was associated with dose-response increases in risks of being underweight and stunted, reflected by weight and length for age being below $2 z$-scores, respectively, at 6 months of age. The risk of wasting $(<-2 z$-scores in weight for length) was only significantly higher for infants of severely food-insecure households, suggesting that underlying determinants were likely to be of a longer than shorter term nature. These findings remain robust when additionally adjusting for intervention arms, excluding teenage mothers 19 years or younger, or excluding children with birth defects (data not shown).

Our cross-sectional associations are consistent with many, though not all, studies among different aged children using a similar HFI scale in resource-limited environments ${ }^{(26-28)}$. A pooled analysis of data from four South Asian, two SubSaharan Africa and two Latin American countries found a $0 \cdot 2$ SD decrease in height-for-age $z$-scores among children aged $2-5$ years for each ten-point score increase in $\mathrm{HFI}^{(26)}$. In Pakistan, infants 6-18 months of age from food-insecure households reporting hunger in the past 12 months were three times more likely to be stunted than children from food-secure homes ${ }^{(27)}$. A dose-response relationship was also documented among preschool children in Colombia ${ }^{(28)}$, where mild, moderate and severe HFI was associated with 28, 58 and $65 \%$ increased odds of stunting and $11(P>0.05), 47$ and $89 \%$ increased odds of being underweight after controlling for demographic and SES factors. Elsewhere in Bangladesh, Saha et al. ${ }^{(29)}$ observed risks of stunting and underweight from 1 to 24 months of age to be lowest in food secure and highest the most food-insecure households. However, in cross-sectional studies in $\mathrm{Nepal}^{(30)}$ and Sri Lanka ${ }^{(31)}$, researchers failed to observe growth faltering in preschoolers from food-insecure homes.

While we observed a linear decline in arm circumference and BMI with each decrement in household food security, risk of wasting below $-2 z$-scores was increased only within severely insecure homes, as found elsewhere in Bangladesh ${ }^{(29)}$ but not in other countries ${ }^{(26,28,30)}$. A less consistent association with child wasting suggests that food insecurity, as classified by perceptionbased questions, may be more strongly representing long-term than acute food deprivation ${ }^{(32)}$.

A unique feature of our study was its prospective design that enabled us to identify and partition, through a stepwise procedure, effects of potential maternal mediators of the HFIinfant malnutrition association. In rural Bangladesh, women may compromise their own energy intake ${ }^{(33,34)}$ and dietary diversity ${ }^{(35)}$ to ensure adequacy of diet for their husbands and children. Furthermore, a clear linkage has been made between HFI and dietary quality and energy intake of women in poor societies $^{(5-7)}$, suggesting maternal nutritional status may be a sensitive indicator of HFI. Poor maternal nutritional status may also increase the risk of preterm birth and small-for-GA ${ }^{(36)}$; both predict child undernutrition ${ }^{(11)}$. Importantly, we observed that half or more of all infant size deficits linked to post-partum food insecurity were explained by maternal nutritional factors before or during pregnancy, representing a period of a year or more before the 6-month recall period. Specifically, maternal height, reflecting, in part, long-term nutritional consequence ${ }^{(37)}$, explained a $23-30 \%$ and $28-38 \%$ of the food security-related weight and length deficit of infants at 6 months of age. While mechanisms remain poorly understood, shorter maternal stature is a known contributor to smaller birth size and increases risk of infant and childhood malnutrition ${ }^{(32)}$. Maternal arm circumference explained additionally approximately $14-21 \%$ of an infant's unadjusted weight and $7-11 \%$ of the length deficit associated with HFI, consistent with data that link maternal nutritional status during pregnancy to fetal ${ }^{(38)}$ and postnatal growth $^{(39)}$. Finally, using birth length as an indicator of the adequacy of growth throughout gestation ${ }^{(40,41)}$ and ponderal index to reflect especially late gestation fetal weight gain ${ }^{(42)}$, we estimated that nutritional, hormonal and disease factors regulating these facets of growth explained $17-20 \%$ of the food insecurity-related deficit in weight and $11-20 \%$ of the associated deficit in length at 6 months of age, independent of maternal nutritional status near the outset of pregnancy. Supported by previous antenatal food ${ }^{(43)}$ and micronutrient supplementation interventions ${ }^{(44)}$, findings from our study also emphasise the importance to correct food insecurity during pregnancy to reduce malnutrition in fetal period as well as in early childhood.

Postnatal breast and complementary feeding practices coupled with recorded morbidity experiences recorded during the actual recall period accounted for small (6-13 and 4-7\%, respectively) and non-significant fractions of the infant growth deficits in most anthropometric measures. Maternal and household SES factors accounted for virtually all of the remaining infant growth deficits were associated with levels of HFI, albeit minor remaining decrements in our statistical models. Interestingly, another study also failed to find child dietary diversity mediating the relationship between HFI and preschool child undernutrition in Bangladesh, Ethiopia and Vietnam $^{(45)}$. The authors speculated that the strong associations 
between HFI and adequacy of child size may be explained by maternal wasting and undernutrition before and during pregnancy, as suggested by the findings in the present, large prospective study.

Our study is strengthened by a large sample size and a considerably large number of measures of nutritional, health and behavioural variables in mother-infant dyads. Also, our statistical analyses demonstrate the influence of a variety of factors on early infant growth. Some limitations should be noted. We assume the HFI measured at 6 months postpartum represents the chronic condition in our study area. The HFI status may vary from early pregnancy to 6 months postpartum, although the maternal dietary diversity measured in early pregnancy, late pregnancy and 3 months postpartum seemed to consistently decrease with HFI measured at 6 months postpartum in our sample ${ }^{(7)}$. We believe the chronic HFI assumption is likely valid, at least at population level, given the evidence from other studies measuring food insecurity repeatedly in similarly resource-poor settings ${ }^{(14,46)}$. Preterm birth was not confirmed by ultrasound, but the overall preterm birth prevalence estimated in our sample (18\%) was comparable to the pooled estimate for Bangladesh $(19 \%)^{(47)}$. Additionally, postnatal factors, such as feeding practices and morbidity, were self-reported, and measurement errors may have occurred. Residual confounding due to unmeasured variables is also possible. Future studies are needed to explore whether the HFI-child growth relationship persist beyond 6 months of age.

\section{Conclusion}

In this rural South Asian setting, HFI was associated with small infant size, which appeared to be largely acting through a maternal-fetal nutrition pathway. Our findings also suggest that maternal responses about recent food insecurity may be expected to reflect a far longer period of perceived stress. Policies seeking to alleviate potential consequences of food insecurity on infant nutritional status may need to address maternal food deprivation during, and likely before, pregnancy.

\section{Acknowledgements}

We acknowledge all JiVitA project staff who have helped collect data and all participants.

The study was supported by grant GH 614, the 'Global Control of Micronutrient Deficiency', from The Bill \& Melinda Gates Foundation, Seattle, WA, USA, with additional support provided through the Sight and Life Global Nutrition Research Institute, Baltimore, MD, USA.

$\mathrm{M}$. N. formed the research question, conducted the literature review, analysed and interpreted the data, and prepared the first draft of the manuscript. A. A. S., S. M., A. L., H. A., S. S., R. K., P. C. and K. P. W. Jr designed and conducted the parent trial. L. S.-F. W. managed data sets and had full access to the data used in this study. K. P. W. Jr contributed to data analysis, interpretation and manuscript preparation. M. N. had primary responsibility for final content. All authors read and approved the final manuscript.

The authors declare that there are no conflicts of interest.

\section{References}

1. De Pee S, Brinkman H-J, Webb P, et al. (2009) How to ensure nutrition security in the global economic crisis to protect and enhance development of young children and our common future. J Nutr 140, 138S-142S.

2. FAO, International Fund for Agricultural Development, UNICEF, et al. (2019) The State of Food Security and Nutrition in the World 2019. Safeguarding Against Economic Slowdowns and Downturns. Rome: FAO.

3. National Institute of Population Research and Training, Mitra and Associates, ICF International (2016) Bangladesh Demographic and Health Survey 2014. Dhaka, Bangladesh, and Rockville, MD: NIPORT, Mitra and Associates, and ICF International.

4. Bellamy C (1998) The State of the World's Children 1998: Focus on Nutrition. Oxford and New York: Oxford University Press for UNICEF.

5. Kendall A, Olson CM \& Frongillo EA Jr (1996) Relationship of hunger and food insecurity to food availability and consumption. J Am Dietetic Assoc 96, 1019-1024.

6. Tarasuk VS \& Beaton GH (1999) Women's dietary intakes in the context of household food insecurity. J Nutr 129, 672-679.

7. Na M, Mehra S, Christian P, et al. (2016) Maternal dietary diversity decreases with household food insecurity in rural Bangladesh: a longitudinal analysis. J Nutr 146, 2109-2116.

8. Kramer MS (1987) Determinants of low birth weight: methodological assessment and meta-analysis. Bull World Health Organ 65, 663.

9. Neufeld LM, Haas JD, Grajéda R, et al. (2004) Changes in maternal weight from the first to second trimester of pregnancy are associated with fetal growth and infant length at birth. Am J Clin Nutr 79, 646-652.

10. Muslimatun S, Schmidt MK, West CE, et al. (2002) Determinants of weight and length of Indonesian neonates. Eur J Clin Nutr 56, 947.

11. Christian P, Lee SE, Donahue Angel M, et al. (2013) Risk of childhood undernutrition related to small-for-gestational age and preterm birth in low- and middle-income countries. Int J Epidemiol 42, 1340-1355.

12. Frith AL, Naved RT, Persson LA, et al. (2012) Early participation in a prenatal food supplementation program ameliorates the negative association of food insecurity with quality of maternal-infant interaction. J Nutr 142, 1095-1101.

13. Webb-Girard A, Cherobon A, Mbugua S, et al. (2012) Food insecurity is associated with attitudes towards exclusive breastfeeding among women in urban Kenya. Matern Child Nutr $\mathbf{8}$, 199-214.

14. Saha KK, Frongillo EA, Alam DS, et al. (2008) Household food security is associated with infant feeding practices in rural Bangladesh. J Nutr 138, 1383-1390.

15. Cook JT, Frank DA, Berkowitz C, et al. (2004) Food insecurity is associated with adverse health outcomes among human infants and toddlers. J Nutr 134, 1432-1438.

16. Campbell AA, de Pee S, Sun K, et al. (2009) Relationship of household food insecurity to neonatal, infant, and under-five child mortality among families in rural Indonesia. Food Nutr Bull 30, 112-119.

17. West KP, Shamim AA, Mehra S, et al. (2014) Effect of maternal multiple micronutrient vs iron-folic acid supplementation on infant mortality and adverse birth outcomes in rural Bangladesh: the JiVitA-3 randomized trial. JAMA 312, 26492658.

18. Labrique $\mathrm{AB}$, Christian $\mathrm{P}$, Klemm RD, et al. (2011) A clusterrandomized, placebo-controlled, maternal vitamin A or betacarotene supplementation trial in Bangladesh: design and methods. Trials 12, 102. 
19. Zerfas AJ (1975) The insertion tape: a new circumference tape for use in nutritional assessment. Am J Clin Nutr 28, $782-787$.

20. Kennedy G, Razes M, Ballard T, et al. (2010) Measurement of dietary diversity for monitoring the impact of food based approaches. In International Symposium on Food and Nutrition Security: Food-based Approaches for Improving Diets and Raising Levels of Nutrition, 7-9 December 2010, Rome, Italy [B Thompson and L Amoroso, editors]. Boston, MA: FAO/CABI.

21. Gunnsteinsson S, Labrique AB, West KP Jr, et al. (2010) Constructing indices of rural living standards in Northwestern Bangladesh. J Health Popul Nutr 28, 509.

22. Christian P, Klemm R, Shamim AA, et al. (2012) Effects of vitamin $A$ and $\beta$-carotene supplementation on birth size and length of gestation in rural Bangladesh: a cluster-randomized trial. Am J Clin Nutr 97, 188-194.

23. World Health Organization (2010) Indicators for Assessing Infant and Young Child Feeding Practices: Part 2: Measurement. Geneva: WHO.

24. Coates J, Webb P \& Houser R (2003) Measuring Food Insecurity: Going Beyond Indicators of Income and Anthropometry. Washington, DC: Food and Nutrition Technical Assistance Project, Academy for Educational Development.

25. Shamim AA, Kabir A, Merrill RD, et al. (2013) Plasma zinc, vitamin $\mathrm{B}_{12}$ and $\alpha$-tocopherol are positively and plasma $\gamma$-tocopherol is negatively associated with $\mathrm{Hb}$ concentration in early pregnancy in north-west Bangladesh. Public Health Nutr 16, 1354-1361.

26. Psaki S, Bhutta ZA, Ahmed T, et al. (2012) Household food access and child malnutrition: results from the eight-country MAL-ED study. Popul Health Metr 10, 24.

27. Baig-Ansari N, Rahbar MH, Bhutta ZA, et al. (2006) Child's gender and household food insecurity are associated with stunting among young Pakistani children residing in urban squatter settlements. Food Nutr Bull 27, 114-127.

28. Hackett M, Melgar-Quiñonez H \& Álvarez MC (2009) Household food insecurity associated with stunting and underweight among preschool children in Antioquia, Colombia. Rev Panam Salud Pública 25, 506-510.

29. Saha KK, Frongillo EA, Alam DS, et al. (2009) Household food security is associated with growth of infants and young children in rural Bangladesh. Public Health Nutr 12, 1556-1562.

30. Osei A, Pandey P, Spiro D, et al. (2010) Household food insecurity and nutritional status of children aged 6 to 23 months in Kailali district of Nepal. Food Nutr Bull 31, 483-494.

31. Rannan-Eliya R, Hossain S, Anuranga C, et al. (2013) Trends and determinants of childhood stunting and underweight in Sri Lanka. Ceylon Med J 58, 10-18.

32. Özaltin E, Hill K \& Subramanian S (2010) Association of maternal stature with offspring mortality, underweight, and stunting in low- to middle-income countries. JAMA $\mathbf{3 0 3}$, $1507-1516$.

33. Abdullah M \& Wheeler EF (1985) Seasonal variations, and the intra-household distribution of food in a Bangladeshi village. Am J Clin Nutr 41, 1305-1313.

34. Kramer E, Peterson K, Rogers B, et al. (1997) Intrahousehold allocation of energy intake among children under five years and their parents in rural Bangladesh. Eur J Clin Nutr 51, 750-756.

35. Bouis HE, Eozenou P \& Rahman A (2011) Food prices, household income, and resource allocation: socioeconomic perspectives on their effects on dietary quality and nutritional status. Food Nutr Bull 32, S14-S23.

36. Khanam R, Lee AC, Mitra DK, et al. (2019) Maternal short stature and under-weight status are independent risk factors for preterm birth and small for gestational age in rural Bangladesh. Eur J Clin Nutr 73, 733-742.

37. Silventoinen K (2003) Determinants of variation in adult body height. J Biosoc Sci 35, 263-285.

38. Neggers Y, Goldenberg RL, Cliver SP, et al. (1995) The relationship between maternal and neonatal anthropometric measurements in term newborns. Obstet Gynecol 85, 192-196.

39. Novotny R \& Haas JD (1987) Maternal anthropometry and infant growth with exclusive breast feeding in La Paz, Bolivia. J Trop Pediatr 33, 309-314.

40. Villar J \& Belizan JM (1982) The timing factor in the pathophysiology of the intrauterine growth retardation syndrome. Obstet Gynecol Surv 37, 499-506.

41. Snijders R \& Nicolaides K (1994) Fetal biometry at 14-40 weeks' gestation. Ultrasound Obstet Gynecol 4, 34-48.

42. Walther F \& Ramaekers L (1982) The ponderal index as a measure of the nutritional status at birth and its relation to some aspects of neonatal morbidity. J Perinat Med 10, 42-47.

43. Imdad A \& Bhutta ZA (2012) Maternal nutrition and birth outcomes: effect of balanced protein-energy supplementation. Paediatr Perinat Epidemiol 26, 178-190.

44. Ramakrishnan U, Grant FK, Goldenberg T, et al. (2012) Effect of multiple micronutrient supplementation on pregnancy and infant outcomes: a systematic review. Paediatr Perinat Epidemiol 26, 153-167.

45. Ali D, Saha KK, Nguyen PH, et al. (2013) Household food insecurity is associated with higher child undernutrition in Bangladesh, Ethiopia, and Vietnam, but the effect is not mediated by child dietary diversity. J Nutr 143, 2015-2021.

46. Penny ME, Humphries DL, Woldehanna T, et al. (2015) Crosssectional and longitudinal associations between household food security and child anthropometry at ages 5 and 8 years in Ethiopia, India, Peru, and Vietnam. J Nutr 145, 1924-1933.

47. Chawanpaiboon S, Vogel JP, Moller A-B, et al. (2019) Global, regional, and national estimates of levels of preterm birth in 2014: a systematic review and modelling analysis. Lancet Glob Health 7, e37-e46. 